\title{
Effect of Exercise and Weight Loss in Polycystic Ovarian Syndrome among Obese Women
}

\author{
Thomas Cochrane ${ }^{1}$, Tengku Fadilah Tengku-Kamalden ${ }^{2 *}$, Rachel Davey ${ }^{1}$ and \\ Roxana Dev Omar Dev ${ }^{2}$ \\ ${ }^{1}$ Centre for Research and Action in Public Health, University of Canberra, Bruce ACT 2617, Australia \\ ${ }^{2}$ Faculty of Educational Studies, Universiti Putra Malaysia, Serdang 43400, Selangor, Malaysia
}

\begin{abstract}
Ovulation and fertility can be improved by weight loss in obese women with Polycystic Ovarian Syndrome (PCOS). The aim of this study was to investigate the effectiveness of a twelve-week supervised exercise program in combination with dietary restrictions for obese women with PCOS. The study is a quasi-experimental research and used an experimental pre- and post-test design. Fifteen women recruited from Fertility Clinic, Jessops Hospital for Women, Sheffield took part in this study. Respiratory exchange ratio (RER), heart rate, perceived exertion (RPE), and Houston non-exercise activity code were recorded. Height, weight, and body girth measurements were taken to calculate body mass index, fat percentage, and lean body weight. The intervention group lost an average of $3.1 \mathrm{~kg}$ and gained $3.45 \mathrm{~kg}$ of lean body weight. Loss of fat percentage was $12.1 \%$. No significant difference was found in the control group. The RER and heart rate value decreased for the same workload in the intervention group, indicating higher tolerance towards exercise

ARTICLE INFO

Article history:

Received: 13 November 2020

Accepted: 11 March 2021

Published: 14 April 2021

DOI: https://doi.org/10.47836/pjssh.29.S1.07

E-mail addresses:

t.cochrane@qut.edu.au (Thomas Cochrane)

tengku@upm.edu.my (Tengku Fadilah Tengku-Kamalden)

Rachel.Davey@canberra.edu.au (Rachel Davey)

rdod@upm.edu.my (Roxana Dev Omar Dev)

*Corresponding author intensity. However, the changes for both groups were not significant. The average group compliance rate was 53\% (at least two sessions per week). Bearing in mind the small sample size $(n=4)$ for control, the improvement in fitness, significant weight loss, and body composition change (increase in fat-free mass) was achieved in this study. Twelve weeks of exercise, combined with dietary advice, were sufficient to benefit PCOS obese women. The research has
\end{abstract}

ISSN: 0128-7702

e-ISSN: 2231-8534 
achieved a commendable weight-loss objective and has demonstrated increases in standards of fitness among obese women.

Keywords: Dietary restriction, exercise, fertility, obesity, weight loss

\section{INTRODUCTION}

According to a WHO (2020), in both developed and developing countries, obesity is increasing globally at an unprecedented pace. This is a matter of concern because, for certain severe and morbid conditions, such as diabetes mellitus, hypertension, dyslipedemia, coronary heart disease and some cancers, obesity raises risks (Park et al., 2018). The risk of death due to coronary heart disease and diabetes mellitus is also increased (de Melo et al., 2017). Obesity in women may predispose to disorders like hirsutism (excessive hair growth on face and chest), virilization (production of masculine secondary sex changes in the female including voice changes and development of male type baldness), oligomenorrhea (reduced frequency of periods), and polycystic ovarian syndrome (PCOS), an endocrine abnormality. It is also a cause of anovulatory (failure to ovulate) infertility (Chandler, 2018).

A common endocrine disorder is Polycystic Ovary Syndrome (PCOS) in women of reproductive age (Ortega et al., 2016). PCOS is a condition characterized by disruption of the normal processes leading to ovulation and associated with hyperandrogenism, normal or elevated oestrogen levels (Biswas et al., 2002).
In PCOS obese women, there was an increase in circulating total oestrogen (higher level of oestrone than oestradiol). A higher aromatase activity (which converts androgens to oestrogen) and high oestrone levels triggers abnormal gonadotropin release. This may generate a wrong feedback signal that can modify the amplitude and pulsation of luteinizing hormones (LH) and suppressing follicle-stimulating hormones (FSH) (Patel \& Shah, 2018). These chain reactions will result in a decrease or absence of the menstrual cycle, thus reducing the chance of getting pregnant for obese women. Scott et al. (2017) also reported that there could be an abnormal regulation of growth hormone $(\mathrm{GH})$ secretion in PCOS women independent of body weight. This could explain LH and FSH stimulation irregularities because GH is responsible for the stimulation of both hormones. An early study by Kannan et al. (2019) showed that obese women made up to $43 \%$ of the women affected by menstrual disturbances, miscarriages, and infertility.

Weight loss through exercise and a sensible diet has been highly recommended as the first treatment for infertile and obese women (Dodd et al., 2011). Women (BMI >30) who have lost an average of $6.3 \mathrm{~kg}$ resumed ovulating and had $80 \%$ chances of getting pregnant. Through exercise, these women not only achieve pregnancy but are healthier than when they started and have a more positive attitude to life. These women did not have to reach their ideal weight to start ovulation, and this can be explained by differences in body 
fat distribution (Goodarzi et al., 2011). An average weight loss of $10 \%$ was found to improve many abnormal hormonal parameter characteristics of PCOS. LH, FSH, and Testosterone return to normal levels, and ovulation is resumed. However, despite an increase in SHBG, it will still be surpassed if the subject is still obese (Kaaks et al., 2002).

Exercise programs for weight loss vary in intensity, frequency, and duration, causing significant discrepancies among research results (Kamalden \& Gasibat, 2020; Samsudin et al., 2019; Safikhani et al., 2011a, 2011b; Safikhani, 2010;) . Accelerated weight loss is not necessarily the result of exercise and dietary restrictions (Park et al., 2019). It was also suggested that exercise would have a more profound effect on men than women due to adipose tissue distribution (Despres et al., 1991). Obese women with adipose tissue (android) distribution of the male type responded to exercise essentially like males. Women with female type adipose tissue (gynoid) were found to have no metabolic improvements after training for three months, although insulin insensitivity improved (Pattanakuhar et al., 2017). Even so, physical activity was still suggested to stabilize hormonal imbalance, but it is expected to induce different responses in catecholamine and insulin metabolism in obese subjects as compared to subjects with average body weight (Belfort-DeAguiar \& Seo, 2018). Hyperinsulinemia and insulin resistance, standard features of women with PCOS, were suggested to be influenced by body fat distribution. These are also associated with a higher frequency of menstrual abnormalities (Conway et al., 2016).

Exercise assists in balancing energy expenditure and calorie intake. There are three categories of energy expenditure: the resting metabolic rate (RMR), the thermal effect of a meal (TEM), and the thermal effect of activity (TEA). All three have been shown to decrease with low-calorie intake except TEM in the obese subject. This could be due to the defective wastage component metabolism, which leads to a surplus of calories (MacKenzie-Shalders et al., 2020). Exercise can increase RMR, TEM, and TEA. The dietary reduction will decrease fat mass (FM) as well as fat-free mass (FFM). However, exercise helps retain or increase FFM while depleting FM. FFM has high metabolic rate characteristics and is essential for maintaining skeletal integrity to prevent and reduce osteoporosis (Sarkar et al., 2015). Loss of FFM may be deleterious by decreasing resting metabolism, resulting in weight management difficulties (Chaston et al., 2007).

So it has been suggested that reducing caloric intake and increasing physical activity will maximize weight loss. Besides, exercise was said to suppress appetite during intense activity due to the increase in catecholamines and physical activity's thermic effect. However, Wing (2004) found that a combination of diet and exercise in women did not give any extra weight loss of fat loss as compared to dieting alone. Whichever the case is, in developing an exercise program for the obese, the aim is 
to increase RMR. However, the weight loss program's effectiveness will vary according to each individual depending on body fat distribution, self-motivation and peer encouragement.

Respiratory exchange ratio (RER) can be used to indicate the body's substrate utilization of energy and the presence of lactic acid (Nakano et al., 2019). During regular activity, a mixture of the nutrient is usually used. This includes carbohydrates, fat, and a small percentage of protein. The RER value is between 0.7 and 1.00. A value of 0.8 can be assumed as a result of 1 shows that majority of the energy is catabolized from carbohydrates while a value of 0.7 indicates metabolism from fat (Ong et al., 2018). This study aimed to determine the effectiveness of a twelve-week supervised exercise program combined with dietary advice for weight loss in PCOS obese women.

\section{MATERIALS AND METHODS}

\section{Study Design}

As a quasi-experimental, pre-test/post-test study, subjects were not randomly selected for treatment and control groups. This is an experiment in which measurements are taken both before and after treatment. Design means that you can see the effects of some type of treatment on a group (Mukherjee, 2019). The subjects were not randomly allocated to groups. Subjects from the exercise group were living near Sheffield and could attend the supervised sessions.

The University's Ethics Committee certified that the study was accomplished according to the Declaration of Helsinki. The $\mathrm{G} *$ Power software version 3.1 was used to determine the sample size (Bujang \& Baharum, 2017). The following parameters were selected: large effect size $(f=0.85)$, an alpha level of .05 , a power level of 0.8 , and two groups. The total sample size was determined to be at least 12 . However, the total sample size of this study was 15. Participants in this study included 15 females, recruited from the Fertility Clinic, Jessop Hospital for Women, who were an average of $30.1 \pm 4.6$ years of age for intervention group and $37.5 \pm 4.0$ of the control group (Table 1). All participants met the inclusion criteria which included being a member of the recruited from the mentioned fertility center. Thus, 15 females were purposefully selected to participate in this study (11 interventions and four controls). The subjects were all diagnosed as having Polycystic Ovarian Syndrome (PCOS) and were considered obese $\left(\mathrm{BMI}>28.6 \mathrm{~kg} / \mathrm{m}^{2}\right)$. Subjects were not receiving or consuming any hormonal medication, sedentary, and had been screened for risk factors (high blood pressure and cardiac problems). Subjects were advised on their dietary intake by a qualified nutritionist at the beginning and during the study. Therefore, the present study is based on a pre-test/post-test design without random assignment, or what is commonly called a "non-equivalent control group design"(Shadish et al., 2002).

For the control group, subjects were recruited from the same clinic. Eight subjects in the control group were living away from Sheffield and unable to attend the 
Table 1

Mean + (standard deviation) for physical activity and anthropometric measurements. (NS = non-significant; $p>0.05)$

\begin{tabular}{llll}
\hline Characteristics & $\begin{array}{l}\text { Intervention Group } \\
(\mathrm{n}=11)\end{array}$ & $\begin{array}{l}\text { Control Group } \\
(\mathrm{n}=4)\end{array}$ & P-value \\
\hline Age & $30.1 \pm 4.6$ & $37.5 \pm 4.0$ & $\mathrm{P}<0.05$ \\
Height & $1.61 \pm 0.06$ & $1.58 \pm 0.07$ & $\mathrm{NS}$ \\
Weight & $92.95 \pm 15.63$ & $85.62 \pm 13.46$ & $\mathrm{NS}$ \\
$\begin{array}{l}\text { Body Mass Index } \\
(\mathrm{kg} / \mathrm{m} 2)\end{array}$ & $35.94 \pm 5.69$ & $34.35 \pm 5.34$ & $\mathrm{NS}$ \\
$\begin{array}{l}\text { Houston Non- } \\
\begin{array}{l}\text { Exercise Activity } \\
\text { Code }\end{array}\end{array}$ & $2 \pm 1.4$ & $1.5 \pm 1$ & $\mathrm{NS}$ \\
$\begin{array}{l}\text { Estimated } \mathrm{VO}_{2} \text { max } \\
(\mathrm{ml} / \mathrm{kg} / \mathrm{min})\end{array}$ & $22.47 \pm 4.96$ & $19.06 \pm 4.69$ & $\mathrm{NS}$ \\
\hline
\end{tabular}

supervised exercise session. To minimize the contamination, the researcher asked the patient to minimize physical activity and maintain their condition at the time for a 'waiting period' of twelve weeks. Subjects were sent the full detail of the study, an information sheet, a map of directions, and an appointment letter for a treadmill test at the beginning of the study. Informed consent was obtained from subjects before the study. Subjects were encouraged to ask questions and reassured their rights to withdraw from the study.

\section{Instruments and Procedures}

Initial pilot work was undertaken to determine whether a modified Balke protocol would be suitable for these subjects. A Balke protocol is a continuous type maximal exercise treadmill test suitable for sedentary men and women (Kang et al., 2001). The Balke protocol maintains a constant speed (3.3mph) but increases grade by $1 \%$ each minute (Balke \& Ware, 1959). Balke justified his concern with physical fitness due to the alterations which were occurring with the onset of work automation, in the means of transportation and in many daily activities, leading to significant reduction of effort and consequently of physical exercise performance (Beltz et al., 2016). The initial workload was $5.0 \mathrm{~km} / \mathrm{h}$ at $0 \%$ grade for warm-up before it increased to $5.5 \mathrm{~km} / \mathrm{h}$ with an increase of $1 \%$ grade per minute.

The modified protocol took into consideration the fitness level of the subjects as compared to normal subjects. It was necessary to establish a suitable protocol to ensure that it will be strenuous enough to achieve $60-70 \% \mathrm{VO}_{2}$ max without high dropout rates. Four volunteers were used to determine the Balke protocol's suitability and found that a slower speed $(4.5 \mathrm{~km} / \mathrm{h})$ as an initial workload as a warm-up and 
adjusting to treadmill walking might be more appropriate for the potential subjects before increasing it to $5.0 \mathrm{~km} / \mathrm{h}$. The modified Balke protocol was deemed better because it starts at a slower pace, and only changes in grading after a suitable pace has been set, allowing the subjects to adjust comfortably to the treadmill speed.

\section{Outcome Measures and Data Collection}

The pre-intervention baseline data for both groups were collected three weeks before the commencement of exercise classes. These tests were repeated for 12 weeks. All data collection, anthropometric, and physiological tests were conducted in Physiology Laboratory, Sheffield Institute of Sports Medicine and Exercise Science at the Physiotherapy Department of The Royal Hallamshire Hospital. Subject's activity levels were determined using the Houston Activity Code Questionnaire (Jackson et al., 1990). $\mathrm{VO}_{2}$ max values were then predicted for each subject using the formula:

$$
\begin{aligned}
& \mathrm{VO}_{2} \max =56.363+(1.921 \times \text { activity } \\
& \text { level })-(0.381 \times \text { age })-(0.754 \times \text { Body } \\
& \text { Mass Index })
\end{aligned}
$$

The subject's height and weight were also measured to calculate Body Mass Index (BMI). This was measured before the treadmill testing at the beginning and the end of the study. BMI is defined as the ratio of body weight $(\mathrm{kg})$ to the square of height (m).

The body circumference measurements were chosen because of its simplicity and suitability in predicting fatness in obese.
This method was based on the criterion value of body density and percentage of body fat determined by hydrostatic weighing or water displacement methods (Kardani \& Rustiawan, 2020). Once these circumference measurements have been obtained using a cloth tape, corresponding constants A (abdomen), B (right thigh), and $\mathrm{C}$ (right calf or forearm) were determined. Weight of fat and lean body weight was then calculated for comparison according to the following steps:

An initial test was carried out to determine the repeatability of the respiratory exchange ratio (RER) measurement of the gas analyzer. One volunteer, not physically active, carried out the whole protocol four times within a week to avoid the training effect. RER and heart rate were recorded. The standard deviation for the RER is 0.024 , and the heart rate is 0.99 . The standard deviation value above this value indicates changes in measurements.

Calibration of the oxygen $\left(\mathrm{O}_{2}\right)$ and carbon dioxide $\left(\mathrm{CO}_{2}\right)$ analyzer, Cardiopulmonary Exercise Testing Med Graph System (CPX/D) was carried out on each occasion before the testing. This involves setting the right temperature, humidity, and pressure of the occasion. The CPX/D was calibrated against a reference gas sample of $\mathrm{O}_{2}$ and $\mathrm{CO}_{2}$ for every subject. During the treadmill test, subjects wore a mouthpiece and nose clip. The mouthpiece was connected to the breath analyzer, $\mathrm{CPX} / \mathrm{D}$, to obtain a respiratory exchange ratio (RER). RER is the ratio of metabolic exchange. It is the ratio of $\mathrm{CO} 2$ produced 
and $\mathrm{O} 2$ consumed. RER can indicate the substrate being catabolized for energy. At the same time, measurements of ventilator expiration (VE), $\mathrm{VO}_{2}, \mathrm{VCO}_{2}$ were recorded as well at 30 seconds intervals. Heart rate was recorded using the POLAR heart rate monitor (POLAR Sportstester, Finland). Through the exercise test, subjects were asked to indicate their exertion using the Borg rate of perceived exertion (RPE) 10-point Scale.

\section{Treatment for Groups}

Intervention Group-Exercise Group. The exercise classes include three types of activities; aerobic, aquaerobic, and gym sessions, and were conducted over twelve weeks by a qualified instructor and the researcher. The subjects were requested to attend at least two, one hour sessions of supervised exercise classes and were encouraged to do other exercises on their own. Exercise sessions, intensity, and duration were recorded in an exercise diary. Attendance was monitored, and a $50 \%$ attendance was set as a level for compliance (a minimum of 12 classes). The exercise programs involved aerobic exercise (i.e., those involving the oxygen energy system) and aimed to work for the significant muscles group. The researcher was present at all sessions to encourage and co-supervise the exercise session. Subjects were encouraged to drink as often as possible during the exercise sessions to keep them hydrated.
Control Group. Subjects were asked to carry out their regular activity. These subjects were those who do not participate at all in any supervised exercise sessions. Daily activities like walking and gardening were excluded. The subjects were given the same dietary advice as to the intervention group at the beginning of the study.

\section{Statistical Analysis}

Statistical analysis was conducted using SPSS. The groups' baseline characteristics were compared using non-parametric analysis (Mann-Whitney U test). The group pre- and post- measurement of body weight, BMI, PER, heart rate, and other fitness parameters, i.e., RPE and $\mathrm{VO}_{2}$ max, were evaluated using the Wilcoxon Signed-Rank match paired test, to test for differences in median group score. A significance level of $5 \%$ was applied through this study.

\section{RESULTS}

\section{Baseline Measurements}

Physical Activity Level and Anthropometric Measurements. The comparison of baseline measurements between the intervention and control group are displayed in table 1.

All data were compared using Mann Whitney U-Test for independent samples. The control group was significantly older than the intervention group $(p<0.05)$. There were no other significant differences between the other group characteristics. 


\section{Pre/post Measurements}

\section{Pre/post Anthropometric Measurements.}

All data pre-and post-intervention were compared using the Wilcoxon signed-rank test for the intervention and control group see (Table 2). A comparison between groups was made using the Mann Whitney U test. Weight, body mass index and body fat percentage decreased significantly in the intervention group $(\mathrm{p}<0.05)$. There was also a significant increase in the lean body weight of the intervention group. There were no significant changes in the measurements for the control group.

Pre/Post measurements of physical Activity, $\mathrm{VO}_{2}$ max, and Rate of Perceived Exertion.
The pre- and post-intervention data for both groups were calculated using Wilcoxon Signed ranks test for paired samples. Each subject determined RPE values at the end of a treadmill protocol. The intervention group showed a significant increment for the activity codes and $\mathrm{VO}_{2}$ $\max (p<0.05)$. The Mann Whitney U test for independent samples was used to compare the measurements between both groups. No significant difference was found.

\section{Pre/post Measurements of Respiratory Exchange Ratio (RER) and Heart Rate.}

The pre/post-intervention data for both groups' data were compared using Wilcoxon Signed ranks test for paired samples, while the Mann Whitney U test for unpaired samples was used to compare between groups. There were no significant differences

Table 2

Mean (+ SD) Pre/post anthropometric measurement for intervention $(n=9)$ and control group $(n=4)$

$(N S=$ non-significant; $p<0.05)$

\begin{tabular}{lllll}
\hline $\begin{array}{l}\text { Intervention } \\
\text { Group }\end{array}$ & Pre & Post & Difference $(\%)$ & $\mathrm{p}$-value \\
\hline Weight & $89.61 \pm 15.24$ & $86.56 \pm 14.57$ & $-3.1(3.4 \%)$ & $\mathrm{P}<0.01$ \\
$\begin{array}{l}\text { Body Mass } \\
\text { Index (kg/m2) }\end{array}$ & $35.06 \pm 5.87$ & $33.87 \pm 5.66$ & $-1.19(3.4 \%)$ & $\mathrm{P}<0.05$ \\
$\begin{array}{l}\text { \%fat } \\
\text { Lean Body }\end{array}$ & $33.58+/-6.77$ & $28.29+/-7.69$ & $-4.62(12.1 \%)$ & $\mathrm{P}<0.01$ \\
$\begin{array}{l}\text { Weight } \\
\text { Control Group }\end{array}$ & Pre & $58.74 \pm 4.10$ & $+3.45(5.9 \%)$ & $\mathrm{P}<0.05$ \\
\hline $\begin{array}{l}\text { Weight }(\mathrm{kg}) \\
\text { Body Mass }\end{array}$ & $86.53 \pm 13.46$ & $83.9 \pm 12.24$ & $-2.63(3 \%)$ & $\mathrm{NS}$ \\
$\begin{array}{l}\text { Index }(\mathrm{kg} / \mathrm{m} 2) \\
\text { \%fat }\end{array}$ & $34.35 \pm 5.34$ & $33.85 \pm 5.04$ & $-0.5(1.4 \%)$ & $\mathrm{NS}$ \\
$\begin{array}{l}\text { Lean Body } \\
\text { Weight }\end{array}$ & $52.0 \pm 3.42$ & $53.25 \pm 5.03$ & $+1.25(2.3 \%)$ & $\mathrm{NS}$ \\
\hline
\end{tabular}


in measurements for both groups.

Mean percentage changes in RER and heart rate after 12 weeks for the intervention $(n=9)$ and the control group $(n=4)$ were calculated and displayed. There was a decrease in RER for both groups, with the intervention group showing more significant reduction; mean percentage change $( \pm \mathrm{S}$. E.) $=-2.49( \pm 1.78)$ compared to the control group; mean percentage change $( \pm$ S. E.) $=-0.55( \pm 3.0)$. Analysis of the mean percentage change in heart rate showed that the intervention group's mean heart rate decreased; mean percentage change $( \pm \mathrm{S}$. E. $)=-1.95( \pm 5.6)$ while the control group's mean heart rate increased; mean percentage change $( \pm$ S. E. $)=+7.0( \pm 6.8)$.

\section{DISCUSSION}

\section{Pre/Post Measurements}

\section{Pre/post Anthropometric Measurements.}

There were significant decreases in the weight, BMI, and percentage of body fat in the intervention group $(p<0.05)$. Both groups increased the lean body weight, but only the intervention group's change was significant $(\mathrm{p}<0.05)$. There was an average of $3.1 \mathrm{~kg}(3.3 \%)$ weight loss, a reduction of $12.1 \%$ of fat, and an average increase of 3.45 $\mathrm{kg}$ of lean body weight in the intervention group. This indicated that the subjects' body composition had changed due to the effect of diet and aerobic exercise. The dietary restriction may have helped in maintaining total weight loss.

The exercise also helped maintain lean body weight and further increased the quantity of lean body mass in the intervention group (6.24\%). In this study, we used anthropometric measurements to predict body fat and lean body weight. The error in predicting an individual's body fat using this method is \pm 2.5 to $4.0 \%$ (McArdle et al., 2013). This method was chosen because it has relatively low prediction errors, whilst the measurements easy to take, and measurements are well suited for the obese for determining patterns of fat distribution on the body and changes in fat following weight loss (McArdle et al., 2006).

Recoded exercise research studies on PCOS obese women have demonstrated similar findings. Garrow and Summerbell (1995) had shown that diet alone for a $10 \mathrm{~kg}$ weight loss, the expected free mass (FFM) loss was $2.2 \mathrm{~kg}$ in women. However, with a similar weight loss achieved by exercise and dietary restriction, the loss of FFM was reduced to $1.7 \mathrm{~kg}$. Although there was some weight loss, it was not great. In this study, body composition changes did occur but were also relatively small. Any decrease in body weight was due to body fat loss because lean muscle mass was seen to increase in most subjects. The present study has achieved a weight loss of over 3.3\% in just three months. Thus, in the present group, a weight loss of $5 \%$ or more should be within reach if the exercise program continues for another three months, as will be the case in a follow-on study from the present.

Norman et al. (2007) studied the effect of weight loss by gradual dietary changes and regular exercise for over six months. 
The study emphasized a group approach for the treatment of obesity and infertility. The subject lost an average of $6.3 \mathrm{~kg}$ and showed marked improvement in pregnancy and ovulation rates. In this study, the intervention group's ovulation rates were being monitored by a clinician. However, by the sixth week of the exercise program, one subject had successfully resumed her menstruation while the other began to notice a regular menstruation trend. She had irregular menstruation previously. The magnitude of change in body mass and composition is also highly dependent on the exercise program's duration, frequency, and intensity. It may be possible that a threshold exists before alterations in body mass, and composition can occur (Wilmore, 1996).

Pre/Post Measurements of Physical Activity, VO2 Max, and Rate of Perceived Exertion (RPE). There was a significant increase in activity code, and hence estimated $\mathrm{VO}_{2} \max (8.79 \%)(\mathrm{p}<0.01)$. The rate of perceived exertion (RPE) also changed significantly in that it signifies that the subjects perceived the latter treadmill test as more comfortable. RPE was found to be more useful in determining exercise intensity than heart rate. A patient or athlete can use RPE to work within a training zone. This training zone has been identified as 50 $80 \% \mathrm{VO}_{2}$ max, $60-90 \%$ maximal heart rate, and it correlates to 5 to 9 (healthy to very strong) on the RPE 10-point scale. Because high-intensity exercise is not tolerated by overweight subjects, keeping within this training zone is essential for the exercise program (Mascarenhas et al., 2016).

RMR has also been associated with the changes in fitness level, $\mathrm{VO}_{2} \max$, and body composition. However, there were discrepancies between VO2 max and RMR because of insufficient sample size and statistical power to detect differences in RMR between groups that vary in aerobic fitness (Brochu et al., 2001). The RMR can be estimated from parameters such as FFM, weight, body surface area, or combination of age, gender, weight, and height (Dugdale et al., 2010). An increase in the proportion of FFM to total body weight may increase RMR. A low rate resting metabolic rate (RMR) for a given body composition was identified as a risk factor for weight gain and obesity and reported in formerly obese individuals with the genetic predisposition for obesity (Heilbronn \& Ravussin, 2003). Nevertheless, because RMR represents between $60 \%$ to $70 \%$ of the total energy expended each day in an active individual who is not training, an increase of only $1 \%$ $-2 \%$ in RMR could have a significant effect on weight regulation over the long term (Dugdale et al., 2010). A cross-sectional comparison of RMR of exercising subjects and non-exercising subjects may be useful in detecting the effects of large differences in maximal aerobic power and training status on RMR; RMR has also been used in fitness level changes between trained and untrained individuals (Brochu et al., 2001).

Gadducci et al. (2017) evaluated the metabolic changes of obese men and women and the effects of exercise and weight loss. It was found that after three months (three 
one hour sessions per week) of physical training, gynoid obese women did not show any metabolic rate changes. The present study did not differentiate the type of female adiposity, which could explain the difficulty of some women in losing weight. This study looks into consideration that the majority of the PCOS obese women who took part were sedentary, Houston non-exercise activity code $(2 \pm 1)$. This study looks into the improvement of fitness level but did not compare it to the average population. The initial aerobic exercise was a low impact to allow adaptation to the exercise program's new physical challenges and avoid premature dropouts.

\section{Pre/Post Measurements of Respiratory Exchange Ratio (RER) and Heart Rate.}

There was an average decrease in the RER and heart rate for both groups. The RER and heart rate values in both groups were taken as an average at minute $13^{\text {th }}$ to be compared. RER was used to indicate the body's substrate utilization for energy and the presence of lactic acid. During the regular activity, a mixture of the nutrient is usually used. This includes carbohydrates, fat, and a small percentage of protein. The RER value is between 0.7 to 1.00 . A value of 0.8 can be assumed due to the metabolism of a mixture of $40 \%$ carbohydrate and $60 \%$ fat. A value of 1 shows that the majority of the energy is catabolized from carbohydrates, while a value of 0.7 indicates metabolism from fat. During exhaustive exercise, lactic acid is produced. In order to maintain the acid-base balance in the blood, sodium bicarbonate will buffer the lactic acid. Moreover, in this process, carbonic acid is formed and is broken down to $\mathrm{CO}_{2}$ and water. The excess $\mathrm{CO}_{2}$ exits from the lungs, thus adding extra $\mathrm{CO}_{2}$ to the quantity that is typically released during energy metabolism.

In some instances, the RER of a fat person on a high carbohydrate diet can also exceed the value 1. Oxygen is released when a carbohydrate diet can also be converted into fat as it is being stored. This extra oxygen can be used in energy metabolism resulting in less atmospheric $\mathrm{O}_{2}$ consumed even though the usual metabolic complement of $\mathrm{CO}_{2}$ is released during energy metabolism. In this study, the obese subjects took time to breathe through the mouthpiece with the nose clip. The researcher had observed that the majority of the subjects were hyperventilating at the beginning of the treadmill protocol. This could have been caused by a high state of arousal or just mechanical inefficiency of walking on a treadmill.

Nevertheless, it may have been the reason for the fluctuations in the RER measurements during the protocol. Whipp and Davis (1984) suggested that obesity was a factor that limited exercise tolerance because it imposed additional stress to ventilation during exercise. This is a direct result of the increased body mass requiring greater energy exchange. The obese women had an increased metabolic cost of breathing because of the interfering chest wall and abdominal obesity and high breathing frequencies. Besides, obese subjects have reduced 'motor efficiency' as a result of 
relatively low mechanical skill. This is because increased tissue mass and bulk hinders specific limb motion directions requiring increased energy to overcome this inertia. Thus, it has been suggested that the role of support and posture to minimize the effect of obesity should be considered to improve exercise tolerance in obese subjects.

Except for one subject who had asthma on the day of the second treadmill test, the subjects from the intervention group could exercise longer before reaching the RER value of 1 . Some subjects completed the whole protocol without reaching the value 1 at all.

There were many variations in the measurements of the RER. As RER considers the rate of $\mathrm{O}_{2}$ uptake and $\mathrm{CO}_{2}$ produced, this makes it susceptible to many confounding factors, psychologically. $\mathrm{CO}_{2}$ can increase from hyperventilating, and this would not reflect the true metabolic demands of a particular situation. This results in a reduction of the average $\mathrm{CO}_{2}$ level in blood. Furthermore, it is not accompanied by a corresponding increase in oxygen consumption resulting in RER value above 1 . The subjects were anxious and apprehensive during the earlier treadmill test. As some were sedentary and seldom exercise, walking on a treadmill for 15 minutes can be a significant ordeal. Walking into a clinical environment can also be intimidating for some and causes some anxiety to others. Motivation does seem to influence the subjects during the treadmill test. Subjects were more motivated and eager to prove that they were much fitter for the second treadmill test. The intervention group had a significantly lower perceived exertion rate, suggesting that the task was more comfortable for the intervention. Before starting the treadmill test, the subjects were asked to relax for at least 20 minutes to get their heart rate stable and as low as possible. However, as soon as the subject was informed that the test was about to begin, the heart rate started to increase, making the initial rest not useful.

Research on RER has shown that individuals with a high resting RER value are more prone to gain fat. Maunder et al. (2018) found that 24-hour RER could affect weight gain in obese men and women. If 24-hour fat oxidation is low relative to carbohydrate oxidation, the excess in energy will be stored mainly as fat. The heart rate values for both the intervention was lower $(2.9 \%)$ in contrast with an increase in the control group. There were many variations as well in heart rate measurements. The POLAR heart rate monitor could not record the heart rate during the treadmill protocol for some of the more obese subjects because of the thick fat layers, which resulted in low signal quality and erratic response. Fortunately, the rate of perceived exertion was also recorded for each subject.

\section{CONCLUSION}

The twelve weeks exercise program combined with dietary advice has shown a significant change in weight and body composition in the intervention group $(p<0.05)$ compared to those in the control 
group. Although there were changes in the RER and heart rate values for both groups, these were not significant. The rate of perceived exertion, activity code, and predicted $\mathrm{VO}_{2}$ max showed significant changes as well as indicating an improvement in the fitness level of the intervention group. The exercise program's compliance rate was moderate, and the mode of exercise program provided was well received. The study had achieved a commendable target of weight loss and had shown improvements in fitness levels.

\section{IMPLICATIONS}

The group's cohesion and support from all involved in the study were an essential component in the exercise program's success. A more controlled, longer-term study to relate and determine the duration of the beneficial effect of exercise and other physiological changes, i.e. hormonal changes, insulin sensitivity in PCOS women is needed. As the global coronavirus (COVID-19) pandemic unfolds, more than $90 \%$ of the U.S. women residents are confined to their homes, with restaurants, shops, schools, and workplaces shut down to prevent the spreading of the disease. A holistic approach to weight loss in PCOS needs to be adopted: one that discusses not only behavioural reform but any possible mental and emotional obstacles to its successful implementation. Only then can we expect to turn the typified vicious weight-gain loop and exacerbated PCOS features into a liberated virtual cycle of successful weight loss and eventual liberation from PCOS shackles.

\section{ACKNOWLEDGEMENT}

The researchers would like to acknowledge the participants from Jessops Hospital who participated in this research conducted as part of Tengku Fadilah Tengku Kamalden's Master dissertation of Sheffield Institute of Sports Medicine and Exercise Science, University of Sheffield.

\section{REFERENCES}

Balke, B., \& Ware, R. W. (1959). An experimental study of physical fitness of Air Force personnel. United States Armed Forces Medical Journal, 10(6), 675-688.

Belfort-DeAguiar, R., \& Seo, D. (2018). Food cues and obesity: Overpowering hormones and energy balance regulation. Current Obesity Reports, 7(2), 122-129. doi:10.1007/s13679-018-0303-1

Beltz, N. M., Gibson, A. L., Janot, J. M., Kravitz, L., Mermier, C. M., \& Dalleck, L. C. (2016). Graded exercise testing protocols for the determination of VO2max: Historical perspectives, progress, and future considerations. Journal of Sports Medicine, 2016, 1-12. doi:10.1155/2016/3968393

Biswas, M., Thackare, H., Jones, M. K., \& Bowen-Simpkins, P. (2002). Lymphocytic hypophysitis and headache in pregnancy. BJOG: An International Journal of Obstetrics \& Gynaecology, 109(10), 1184-1186. doi:10.1111/ j.1471-0528.2002.01158.x

Brochu, M., Tchernof, A., Dionne, I. J., Sites, C. K., Eltabbakh, G. H., Sims, E. A., \& Poehlman, E. T. (2001). What are the physical characteristics associated with a normal metabolic profile despite a high level of obesity in postmenopausal women? The Journal of Clinical Endocrinology \& Metabolism, 86(3), 1020-1025. doi:10.1210/ jcem.86.3.7365

Bujang, M. A., \& Baharum, N. (2017). Guidelines 
of the minimum sample size requirements for Kappa agreement test. Epidemiology, Biostatistics and Public Health, 14(2), 1-10.

Chandler, M. (2018). New thoughts about obesity. Companion Animal, 23(12), 686-695. doi:10.12968/coan.2018.23.12.686

Chaston, T. B., Dixon, J. B., \& O’Brien, P. E. (2007). Changes in fat-free mass during significant weight loss: A systematic review. International Journal of Obesity, 31(5), 743-750. doi:10.1038/ sj.ijo.0803483

Conway, C. C., Starr, L. R., Espejo, E. P., Brennan, P. A., \& Hammen, C. (2016). Stress responsivity and the structure of common mental disorders: Transdiagnostic internalizing and externalizing dimensions are associated with contrasting stress appraisal biases. Journal of Abnormal Psychology, 125(8), 1079-1089. doi:10.1037/ abn0000163

de Melo, L. G. P., Nunes, S. O. V., Anderson, G., Vargas, H. O., Barbosa, D. S., Galecki, P., Carvalho, A. F., \& Maes, M. (2017). Shared metabolic and immune-inflammatory, oxidative and nitrosative stress pathways in the metabolic syndrome and mood disorders. Progress in Neuro-Psychopharmacology and Biological Psychiatry, 78, 34-50. doi:10.1016/j. pnpbp.2017.04.027

Despres, J. P., Pouliot, M. C., Moorjani, S., Nadeau, A., Tremblay, A., Lupien, P. J., Theriault, G., \& Bouchard, C. (1991). Loss of abdominal fat and metabolic response to exercise training in obese women. American Journal of PhysiologyEndocrinology and Metabolism, 261(2), 159167. doi:10.1152/ajpendo.1991.261.2.e159

Dodd, J. M., Turnbull, D. A., McPhee, A. J., Wittert, G., Crowther, C. A., \& Robinson, J. S. (2011). Limiting weight gain in overweight and obese women during pregnancy to improve health outcomes: The LIMIT randomised controlled trial. BMC Pregnancy and Childbirth, 11(1), 1-5.

$$
\text { doi:10.1186/1471-2393-11-79 }
$$

Dugdale, A. H. A., Curtis, G. C., Cripps, P., Harris, P. A., \& Argo, C. M. (2010). Effect of dietary restriction on body condition, composition and welfare of overweight and obese pony mares. Equine Veterinary Journal, 42(7), 600-610. doi:10.1111/j.2042-3306.2010.00110.x

Gadducci, A. V., de Cleva, R., de Faria Santarém, G. C., Silva, P. R. S., Greve, J. M. D., \& Santo, M. A. (2017). Muscle strength and body composition in severe obesity. Clinics, 72(5), 272-275. doi:10.6061/clinics/2017(05)03

Garrow, J. S., \& Summerbell, C. D. (1995). Metaanalysis: Effect of exercise, with or without dieting, on the body composition of overweight subjects. European Journal of Clinical Nutrition, 49(1), 1-10.

Goodarzi, M. O., Dumesic, D. A., Chazenbalk, G., \& Azziz, R. (2011). Polycystic ovary syndrome: Etiology, pathogenesis and diagnosis. Nature Reviews Endocrinology, 7(4), 219-231. doi:10.1038/nrendo.2010.217

Heilbronn, L. K., \& Ravussin, E. (2003). Calorie restriction and aging: Review of the literature and implications for studies in humans. The American Journal of Clinical Nutrition, 78(3), 361-369. doi:10.1093/ajen/78.3.361

Jackson, A., Blair, S. N., Mahar, M. T., Wier, L. T., Ross, R. M., \& Stuteville, J. E. (1990). Prediction of functional aerobic capacity without exercise testing. Medicine and Science Sports and Exercise, 22(6), 863. doi:10.1249/00005768199012000-00021

Kaaks, R., Lukanova, A., \& Kurzer, M. S. (2002). Obesity, endogenous hormones, and endometrial cancer risk: A synthetic review. Cancer Epidemiology and Prevention Biomarkers, 11(12), 1531-1543.

Kang, J., Chaloupka, E. C., Mastrangelo, M. A., Biren, G. B., \& Robertson, R. J. (2001). Physiological 
comparisons among three maximal treadmill exercise protocols in trained and untrained individuals. European Journal of Applied Physiology, 84(4), 291-295. doi:10.1007/ s004210000366

Kannan, S., Srinivasan, D., Raghupathy, P. B., \& Bhaskaran, R. S. (2019). Association between duration of obesity and severity of ovarian dysfunction in rat-cafeteria diet approach. The Journal of Nutritional Biochemistry, 71, 132143. doi:10.1016/j.jnutbio.2019.05.012

Kardani, G., \& Rustiawan, H. (2020). Circumference measurements on body contest athletes in Indonesia. [Paper presentation]. 4th International Conference on Sport Science, Health, and Physical Education (ICSSHPE 2019), Bandung, Indonesia. https://doi.org/10.2991/ ahsr.k.200214.016

MacKenzie-Shalders, K., Kelly, J. T., So, D., Coffey, V. G., \& Byrne, N. M. (2020). The effect of exercise interventions on resting metabolic rate: A systematic review and meta-analysis. Journal of Sports Sciences, 38(14), 1-15. doi:10.1080/0 2640414.2020.1754716

Mascarenhas, L. P. G., Decimo, J. P., Lima, V. A. de, Kraemer, G. de C., Lacerda, K. R. C. de, \& Nesi-França, S. (2016). Physical exercise in type 1 diabetes: Recommendations and care. Motriz: Revista de Educação Física, 22(4), 223-230.

Maunder, E., Plews, D. J., \& Kilding, A. E. (2018). Contextualising maximal fat oxidation during exercise: Determinants and normative values. Frontiers in Physiology, 9, 599. doi:10.3389/ fphys.2018.00599

McArdle, M. A., Finucane, O. M., Connaughton, R. M., McMorrow, A. M., \& Roche, H. M. (2013). Mechanisms of obesity-induced inflammation and insulin resistance: Insights into the emerging role of nutritional strategies. Frontiers in Endocrinology, 4, 1-23. doi:10.3389/ fendo.2013.00052

McArdle, W. D., Katch, F. I., \& Katch, V. L. (2006). Essentials of exercise physiology. Lippincott Williams \& Wilkins.

Mukherjee, S. P. (2019). A guide to research methodology: An overview of research problems, tasks and methods. CRC Press.

Nakano, I., Hori, H., Fukushima, A., Yokota, T., Kinugawa, S., Takada, S., Yamanashi, K., Obata, Y., Kitaura, Y., Kakutani, N., Abe, T., \& Anzai, T. (2019). Enhanced echo intensity of skeletal muscle is associated with exercise intolerance in patients with heart failure. Journal of Cardiac Failure, 26(8), 685-693. doi:10.1016/j.cardfail.2019.09.001

Norman, R. J., Dewailly, D., Legro, R. S., \& Hickey, T. E. (2007). Polycystic ovary syndrome. The Lancet, 370(9588), 685-697.

Ong, S. R., Crowston, J. G., Loprinzi, P. D., \& Ramulu, P. Y. (2018). Physical activity, visual impairment, and eye disease. Eye, 32(8), 12961303. doi:10.1038/s41433-018-0081-8

Ortega, F. B., Lavie, C. J., \& Blair, S. N. (2016). Obesity and cardiovascular disease. Circulation Research, 118(11), 1752-1770.

Park, H. Y., Kim, Y., \& Nam, S. S. (2019). Effects of exercise training at lactate threshold and detraining for 12 weeks on body composition, aerobic performance, and stress related variables in obese women. Journal of Exercise Nutrition \& Biochemistry, 23(3), 22-28. doi:10.20463/ jenb.2019.0019

Park, S. Y., Wilkens, L. R., Maskarinec, G., Haiman, C. A., Kolonel, L. N., \& Marchand, L. L. (2018). Weight change in older adults and mortality: The multiethnic cohort study. International Journal of Obesity, 42(2), 205-212. doi:10.1038/ ijo.2017.188

Patel, R., \& Shah, G. (2018). High-fat diet exposure 
from pre-pubertal age induces polycystic ovary syndrome (PCOS) in rats. Reproduction, 155(2), 139-149. doi:10.1530/rep-17-0584

Pattanakuhar, S., Pongchaidecha, A., Chattipakorn, N., \& Chattipakorn, S. C. (2017). The effect of exercise on skeletal muscle fibre type distribution in obesity: From cellular levels to clinical application. Obesity Research \& Clinical Practice, 11(5), 112-132. doi:10.1016/j. orcp.2016.09.012

Sarkar, M., Bhardwaj, R., Madabhavi, I., \& Khatana, J. (2015). Osteoporosis in chronic obstructive pulmonary disease. Clinical Medicine Insights: Circulatory, Respiratory and Pulmonary Medicine, 9, 1-17.

Scott, D., Harrison, C. L., Hutchison, S., De Courten, B., \& Stepto, N. K. (2017). Exploring factors related to changes in body composition, insulin sensitivity and aerobic capacity in response to a 12-week exercise intervention in overweight and obese women with and without polycystic ovary syndrome. PloS One, 12(8), 1-13. doi:10.1371/ journal.pone.0182412
Shadish, W. R., Cook, T. D., \& Campbell, D. T. (2002). Experimental and quasi-experimental designs for generalized causal inferencel William R. Shedish, Thomas D. Cook, Donald T. Campbell. Houghton Mifflin.

Whipp, B. J., \& Davis, J. A. (1984). The ventilatory stress of exercise in obesity. American Review of Respiratory Disease, 129(2P2), S90-S92. doi:10.1164/arrd.1984.129.2p2.s90

WHO. (2020). Obesity. World Health Organization. https://www.who.int/topics/obesity/en/

Wilmore, J. H. (1996). Increasing physical activity: Alterations in body mass and composition. The American Journal of Clinical Nutrition, 63(3), 456S-460S. doi:10.1093/ajcn/63.3.456

Wing, R. R. (2004). Behavioral approaches to the treatment of obesity. In Bray, G. A \& Bouchard, C. (Ed.), Handbook of obesity: Clinical applications, 2 (pp.147-167). Marcel Dekker Inc. 